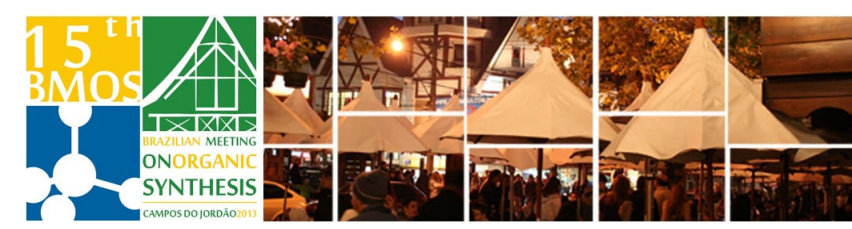

\title{
Brønsted Acid Catalyzed Azlactone Ring Opening With a Wide Range Substratre Scope
}

\author{
Adriane A. Pereira, Pedro P. de Castro, Amanda C. de Mello, Giovanni W. Amarante* \\ Instituto de Ciências Exatas, Departamento de química, Universidade Federal de Juiz de Fora, Brasil \\ *giovanni.amarante@ufff.edu.br
}

Keywords: Azlactone ring, Brønsted acid, organocatalysis

\section{INTRODUCTION}

Azlactones are basically protected amino acids which can be used in the synthesis of natural products. Our research group has been explored the potential of these heterocycles as nucleophiles in the presence of Michael acceptors ${ }^{1}$. In the other hand, it is obvious the presence of two electrophile sites which can react in the presence of different nucleophiles. This strategy is very important since it could be applied in peptide synthesis. Recently, Wang and co-workers described a Brønsted acid as a catalyst for the kinect dynamic resolution following by ring opening of azlactones in the presence of a nucleophile. However, a few azlactone rings and only a special alcohol were well tolerated in the optimized reaction condition. ${ }^{2}$ Thus, in this communication it is showed the (+/-)camphorsulfonic acid (CSA) as effective Brønsted acid catalyst for the azlactone ring opening in the presence of different nucleophiles.

\section{RESULTS AND DISCUSSION}

Our studies raised by reacting the azlactone ring from alanine and octanol in the presence of $10 \mathrm{~mol} \%$ of CSA as catalyst. To our delight the desired product was isolated in $88 \%$ yield (Scheme 1). After optimization of the reaction conditions we found that in fact $10 \mathrm{~mol} \%$ of CSA and DCM was the best condition. Important mention, no reaction was observed without CSA. At this point we evaluated the scope concerning azlactone rings. All of them were well tolerated, for example, a steric bulk azlactone works well, providing the desired product 2 in $82 \%$ yield. By switching the nucleophiles, different alcohols and amines could be used in this reaction condition (Scheme 2). Sugar-based derivatives provided the acyclic products in high yields, but no diastereoselectivity was observed. Secondary alcohol works quite well and even terciary alcohol was well tolerated, giving the desired product in $57 \%$ yield.

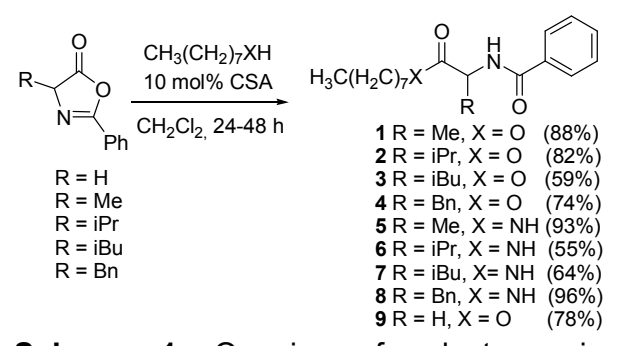

Scheme 1. Opening of azlactone ring by lipophilic alcohols and amines.

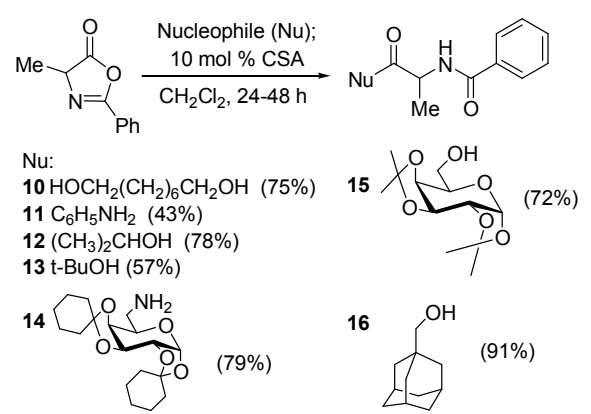

Scheme 2. Opening of azlactone ring by others nucleophiles.

\section{CONCLUSION}

In summary we showed a Brønsted acid as effective organocatalyst for the azlactone ring opening in the presence of different nucleophiles. Several azlactones and lipophilic alcohols/amines were successfully applied, providing the desired products in good to high yields. The development of a chiral catalyst which could be used in the dynamic resolution of the azlactone core is ongoing and will be reported in due course.

\section{ACKNOWLEDGEMENTS}

FAPEMIG, CAPES, UFJF, CNPq and Rede Mineira de Química for financial support.

\section{REFERENCES}

${ }^{1}$ Ávila, E. P.; de Mello, A. C.; Diniz, R.; Amarante, G. W. Eur. J. Org. Chem. 2013, 1881.

${ }^{2}$ Wang, C.; Luo, H. W.; Gong, L. Z. Synlett, 2011, 992. 